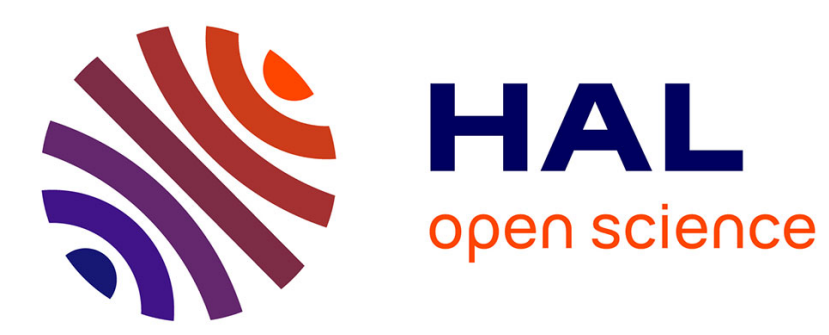

\title{
Caractérisation de Lactuca sativa $L$. et des espèces apparentées par électrofocalisation des estérases
}

Louis Roux, Zhou Chengjiu, Yvette Roux

\section{To cite this version:}

Louis Roux, Zhou Chengjiu, Yvette Roux. Caractérisation de Lactuca sativa L. et des espèces apparentées par électrofocalisation des estérases. Agronomie, 1985, 5 (10), pp.915-921. hal-00884721

\section{HAL Id: hal-00884721 \\ https://hal.science/hal-00884721}

Submitted on 1 Jan 1985

HAL is a multi-disciplinary open access archive for the deposit and dissemination of scientific research documents, whether they are published or not. The documents may come from teaching and research institutions in France or abroad, or from public or private research centers.
L'archive ouverte pluridisciplinaire HAL, est destinée au dépôt et à la diffusion de documents scientifiques de niveau recherche, publiés ou non, émanant des établissements d'enseignement et de recherche français ou étrangers, des laboratoires publics ou privés. 


\section{Caractérisation de Lactuca sativa L. et des espè- ces apparentées par électrofocalisation des esté-} rases.

Louis ROUX, Zhou CHENGJIU $\left(^{*}\right)$ \& Yvette ROUX

I.N.R.A., Laboratoire de Métabolisme et de Nutrition des Plantes, F 78000 Versailles

(*) Faculté Agronomique de Beijing, Beijing. République populaire de Chine

RÉSUMÉ

Les isoenzymes des estérases foliaires de Lactuca sativa et des espèces apparentées ( $L$. aculeata, $L$. serriola, $L$. saligna, $L$. virosa) ont été séparées par électrofocalisation sur gel de polyacrylamide en gradient de pH 4 à 6,5 . La variabilité des estérases est faible au sein de chaque espèce. $L$. sativa et $L$. serriola présentent des diagrammes très voisins qui confirment leur proche parenté ; L. aculeata apparaît proche de $L$. serriola ; L. saligna et $L$. virosa sont très distinctes des trois autres espèces.

Les diagrammes d'estérases permettent de tester le caractère hybride des plantes issues de croisements interspécifiques (L. sativa $\times$ L. virosa).

Mots clés additionnels : Lactuca aculeata, L. saligna, L. serriola, L. virosa.

Isoenzymes of foliar esterases from Lactuca sativa and related species (L. aculeata, L. serriola, L. saligna, $L$. virosa) have been separated by electrofocusing on polyacrylamide gel in a $\mathrm{pH}$ gradient of 4 to 6.5 . Esterases presented a low variability within each species. $L$. sativa and $L$. serriola showed closely related diagrams which confirm their genetic closeness; $L$. aculeata appeared near $L$. serriola, $L$. saligna and $L$. virosa were very distinct from the other three species. The esterase diagrams could be used to test the hybrid character of plants issued from interspecific crosses ( $L$. sativa $\times L$. virosa).

Additional key words : Lactuca aculeata, L. saligna, L. serriola, L. virosa.

\section{INTRODUCTION}

Le genre Lactuca est subdivisé selon FERAKOVA (1976) en 3 sections : Phoenixopus, Mulgedium et Lactuca. A l'intérieur de la section Lactuca, L. sativa L., L. serriola L., L. virosa $\mathrm{L}$. et $L$. saligna $\mathrm{L}$. forment un groupe d'espèces, communes en Europe et au Moyen-Orient, présentant entre elles des affinités particulières : même nombre de chromosomes $2 n=18$, hybridation interspécifique plus ou moins difficile, mais possible (LINDQVIST, 1960a).

Les espèces sauvages apparentées à $L$. sativa présentent un grand intérêt comme réservoir de gènes de résistance à divers agents pathogènes (maladies à virus, Bremia lactucae, ...). L. sativa et $L$. serriola sont 2 espèces très proches par de nombreux caractères ; $L$. virosa et $L$. saligna s'en éloignent nettement (LINDQVIST, 1960a, $b$ ). Une nouvelle espèce, découverte en Israël en 1976, Lactuca aculeata Boiss. \& Ky., serait proche de L. serriola (GLOBERSON et al., 1980). Les croisements interspécifiques entre $L$. sativa et $L$. virosa ou $L$. saligna sont difficiles à réaliser et, dans le cas où l'on obtient une descendance, sa faible fertilité rend difficile la poursuite de la sélection (MAXON SMITH, 1984).

La variabilité des isoenzymes fournit des critères complémentaires du phénotype pour la caractérisation des espèces et cultivars et aussi des marqueurs génétiques utilisables lors des croisements intra ou interspécifiques (MOORE \& COLLINS, 1983). Aucun travail de ce type n'a été réalisé jusqu'ici dans le genre Lactuca. Parmi les systèmes enzymatiques dont la variabilité est généralement élevée figurent les phosphatases acides, les peroxydases et les estérases (GOTTLIEB, 1981 ; JENSEN, 1981). Des essais préliminaires ont montré qu'il était très difficile d'obtenir des diagrammes d'électrofocalisation lisibles de phosphatases acides avec les espèces sauvages du genre Lactuca. Cette difficulté per- 
siste à un degré moindre avec les peroxydases et un travail est en cours à ce sujet. Le présent article est relatif à la variabilité génétique des estérases chez $L$. sativa et les espèces sauvages apparentées ; les estérases ont déjà permis ce type d'étude chez différentes espèces cultivées et les espèces voisines (haricot : BASSIRI \& ADAMS, $1978 a$ et $b$; orge : KAHLER \& ALLARD, 1981 ; SYMEONIDIS \& TSEKOS, 1984 ; tabac : SMITH et al., 1970 ; tomate : TANKSLEY \& RICK, 1980).

\section{MATÉRIEL ET MÉTHODES}

\section{A. Matériel végétal}

Le matériel végétal étudié appartient à 5 espèces différentes :

1 - Variétés cultivées de L. sativa, classées selon les types définis par RODENBURG (1960) :

- laitues à tige : 2 variétés d'origine chinoise,

— laitues grasses : « Sucrine », «Têtue de Nîmes »,

- laitues Batavia : « Calicel », « Cristallo », « Rouge grenobloise ",

- laitues beurre : «Colombus », «Deciminor», " Girelle », « Illera », " Jory », " Melina », " Novir », « Ravel », « Serda », « Verpia », «BZ36».

2 - Lignées issues d'espèces sauvages :

\begin{tabular}{|c|c|c|}
\hline \multirow{3}{*}{ L. serriola } & & origine \\
\hline & Courville & France \\
\hline & LS1 15 & $\gg$ \\
\hline & LS234 & $》$ \\
\hline & LS261 & $\gg$ \\
\hline & CR05 & Norvège \\
\hline & CR07 & Grande-Bretagne \\
\hline \multicolumn{2}{|c|}{ L. aculeata CR09 } & Israël \\
\hline \multirow[t]{6}{*}{ L. virosa } & LS1 84 & France \\
\hline & LS231 & $\gg$ \\
\hline & LS238 & $\gg$ \\
\hline & LS241 & $\gg$ \\
\hline & IVT 1145 & IVT, Hollande (1) \\
\hline & IVT1398 & IVT, Hollande \\
\hline \multirow[t]{3}{*}{ L. saligna } & CR 10 & Israël \\
\hline & CR $16=$ IVT 1346 & IVT, Hollande \\
\hline & CR17 = IVT 1168 & IVT, Hollande \\
\hline
\end{tabular}

3 - Hybrides F1 et F2 entre L. sativa var. "Girelle » (mâle stérile) et $L$. virosa (LS184, LS231, IVT1398).

Ces divers génotypes nous ont été fournis par H. BANnerot et B. Maisonneuve de la Station de Génétique et d'Amélioration des Plantes de Versailles. Ils ont été cultivés soit en serre pendant l'hiver, soit en plein air au printemps.

\section{B. Prélèvement des échantillons de feuilles}

Des échantillons, de poids frais compris entre $0,5 \mathrm{~g}$ et $1 \mathrm{~g}$, sont suffisants : on peut donc faire un prélèvement sur une plante sans la léser de manière irréversible. Le choix des feuilles à prélever est important pour minimiser l'interaction défavorable entre protéines et composés phénoliques des tissus lors de l'extraction des enzymes (RHODES, 1977). Les feuilles âgées,

(1) IVT = Institut voor de Veredeling van Tuinbouwgewassen, Wageningen. notamment chez les espèces sauvages, donnent des diagrammes d'isoenzymes déformés malgré les précautions prises lors de l'extraction des tissus. Il faut prélever de préférence des feuilles proches du point végétatif central lorsque la plante est au stade rosette ou bien, si la plante est en cours de montaison, des feuilles proches des points végétatifs terminaux (axe principal ou axes secondaires). Le fait que la plante soit ou non en cours de montaison n'a pas de répercussion sur la constitution des diagrammes d'estérases. Si le broyage n'est pas effectué dans l'heure qui suit le prélèvement, les échantillons peuvent être conservés au réfrigérateur à $+5^{\circ} \mathrm{C}$ pendant 4 à $5 \mathrm{~h}$.

\section{Obtention des extraits enzymatiques}

\section{Extraction des tissus}

Sauf si l'on est limité par le faible poids d'un échantillon, il est préférable d'éliminer la nervure centrale des feuilles pour faciliter leur broyage. Chaque échantillon est congelé dans un mortier par apport d'azote liquide, puis réduit en poudre à l'aide d'un pilon. La poudre glacée est transférée dans un bécher plongé dans la glace pilée, contenant le tampon d'extraction (Tris- $\mathrm{HCl} 100 \mathrm{mM}$, Dieca $100 \mathrm{mM}$, acide ascorbique $50 \mathrm{mM}$ ) ajusté à $\mathrm{pH} 7,0$ avec $\mathrm{HCl}$. La quantité de tampon utilisée est telle que le broyat final contienne $620 \pm 20 \mathrm{mg}$ de matière fraîche par $\mathrm{ml}$ de broyat.

Après macération pendant 30 à $60 \mathrm{mn}$, le broyat est centrifugé pendant $10 \mathrm{mn}$ à $10000 \mathrm{~g}$ (température $+5{ }^{\circ} \mathrm{C}$ ) dans de petits tubes coniques (type tube Eppendorf). Le surnageant obtenu est centrifugé une nouvelle fois dans les mêmes conditions.

\section{Purification partielle des extraits par filtration sur gel}

L'utilisation d'extraits bruts peut entraîner, particulièrement avec ceux provenant d'espèces sauvages, des artefacts lors de l'électrofocalisation. C'est pourquoi l'ensemble des protéines est séparé des composés de petite masse moléculaire, notamment les composés phénoliques, par filtration sur colonne de gel AcA 202 (gel hydraté constitué de 20 p. 100 d'acrylamide et de 2 p. 100 d'agarose, de marque IBF). Cette purification est réalisée dès la fin du processus d'obtention de l'extrait brut, dans une enceinte refroidie $\left(+5^{\circ} \mathrm{C}\right)$ où l'on peut disposer une batterie de plusieurs colonnes mises en œuvre simultanément (voir tabl. 1).

Le volume d'extrait recueilli $(0,15 \mathrm{ml})$ est inférieur au volume apporté sur la colonne $(0,20 \mathrm{ml})$ ceci afin de ne conserver que la portion centrale, la plus concentrée, du pic d'élution. Les extraits purifiés sont utilisés dans les $24 \mathrm{~h}$ qui suivent leur obtention.

\section{Séparation des estérases par électrofocalisation}

Les estérases sont séparées par électrofocalisation horizontale sur gel de polyacrylamide en gradient de pH de 4 à 6,5 (plaques prêtes à l'emploi : LKB PAGplates), avec un appareil Multiphor (firme LKB). La partie utile du gradient de $\mathrm{pH}$ se situe en fait entre pH 4,0 et $\mathrm{pH} 6,0$ (fig. 2 et 3). Les échantillons sont 
TABLEAU 1

Purification des extraits enzymatiques par filtration sur colonne de gel AcA 202 (IBF).

Purification of enzyme extracts by gel filtration on AcA 202 (IBF).

En période de non-utilisation chaque colonne (1) Equilibrage de chaque colonne avec le est imprégnée du tampon suivant : citrate de tampon d'extraction des tissus dilué 10 fois : sodium $50 \mathrm{mM}$ pH $6,0+\mathrm{NaN}_{3}(40 \mathrm{mg} / 100 \mathrm{ml}$ Tris $\mathrm{HCl} 10 \mathrm{mM}$, Dieca $10 \mathrm{mM}$, ac. ascorbique de tampon)

$5 \mathrm{mM} .5$ passages successifs de $1 \mathrm{ml}$

(3) Elution par le tampon dilué défini en (1): (4) Elimination des substances éventuellement (5) Rinçage final de la colonne avec le a) $0,20 \mathrm{ml}$ - élimine adsorbées sur la colonne. Tampon citrate tampon citrate de conservation (voir ci-

b) $0,15 \mathrm{ml}$ - conservé pour électrofocalisation (voir ci-dessus) $+1 \%$ de Triton X $100: 1 \mathrm{ml}$ dessus) : 5 passages successifs de 1 ml

Colonnes contenant $1 \mathrm{ml}$ de gel humide (Acrylamide $20 \%$, Agarose $2 \%$ ). Diamètre interne $0,5 \mathrm{~cm} ;$ hauteur de gel $5 \mathrm{~cm}$.

TABLEAU 2

Caractéristiques des électrofocalisations en gradient de $p H 4$ à 6,5 .

Features of electrofocusing in a pH gradient of 4 to 6.5 .

Dimensions des plaques de gel

$\mathrm{L} \times 1 \times \mathrm{e}=122 \times 110 \times 1 \mathrm{~mm}$
Milieux d'imprégnation des bandes de liaison Température du circuit de refroidissement : entre le gel et chaque électrode : $\quad+5{ }^{\circ} \mathrm{C}$

Anode : $\mathrm{H}_{3} \mathrm{PO}_{4} 0,5 \mathrm{M}+$ acide glutamique $0,1 \mathrm{M}$

Cathode : $\beta$ alanine $0,1 \mathrm{M}$

Volume maximal de chaque échantillon : $25 \mu \mathrm{l}$ Conditions électriques :

Nombre d'échantillons : 10 à 12 Puissance constante $12,5 \mathrm{~W}$

Voltage $2000 \mathrm{~V}\}$ valeur limites

Intensité $25 \mathrm{~mA}\}$ admissibles
Déroulement de la séparation dans le temps : préfocalisation $30 \mathrm{mn}$

dépôt de l'échantillon sur le gel pendant $30 \mathrm{mn}$ temps complémentaire $3 \mathrm{~h} 30 \mathrm{mn}$

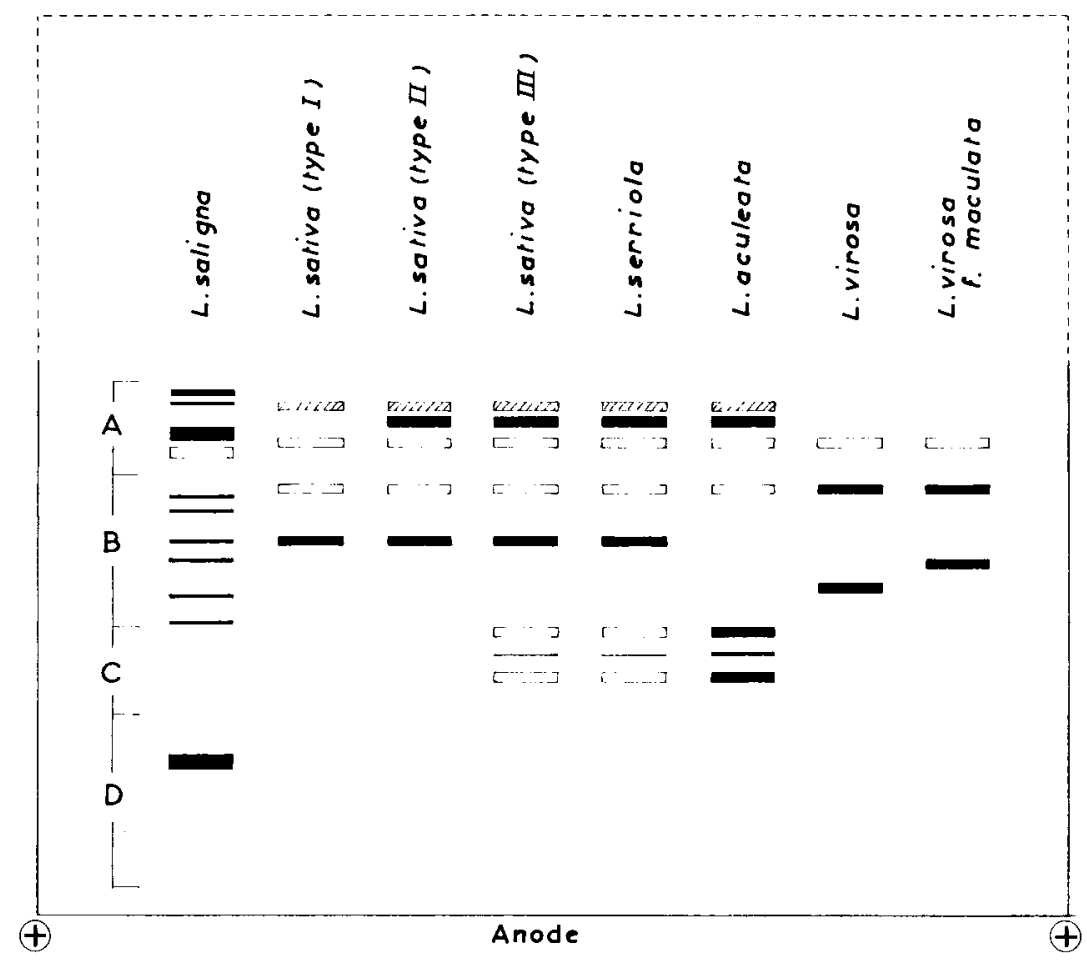

Figure 1

Schéma de la partie anodique des diagrammes d'électrofocalisation (gradient de $\mathrm{pH} 4$ à 6,5) des estérases anodiques foliaires de différentes espèces du genre Lactuca.

Les bandes retenues comme éléments descripteurs sont réparties en 4 zones: $A, B, C$ et $D$.
Scheme of the anodic part of electrofocusing patterns ( $p H$ gradient from 4 to 6.5) of foliar esterases from different Lactuca species. Bands retained as descriptive elements are distributed within 4 zones: $A, B, C$ and $D$. 


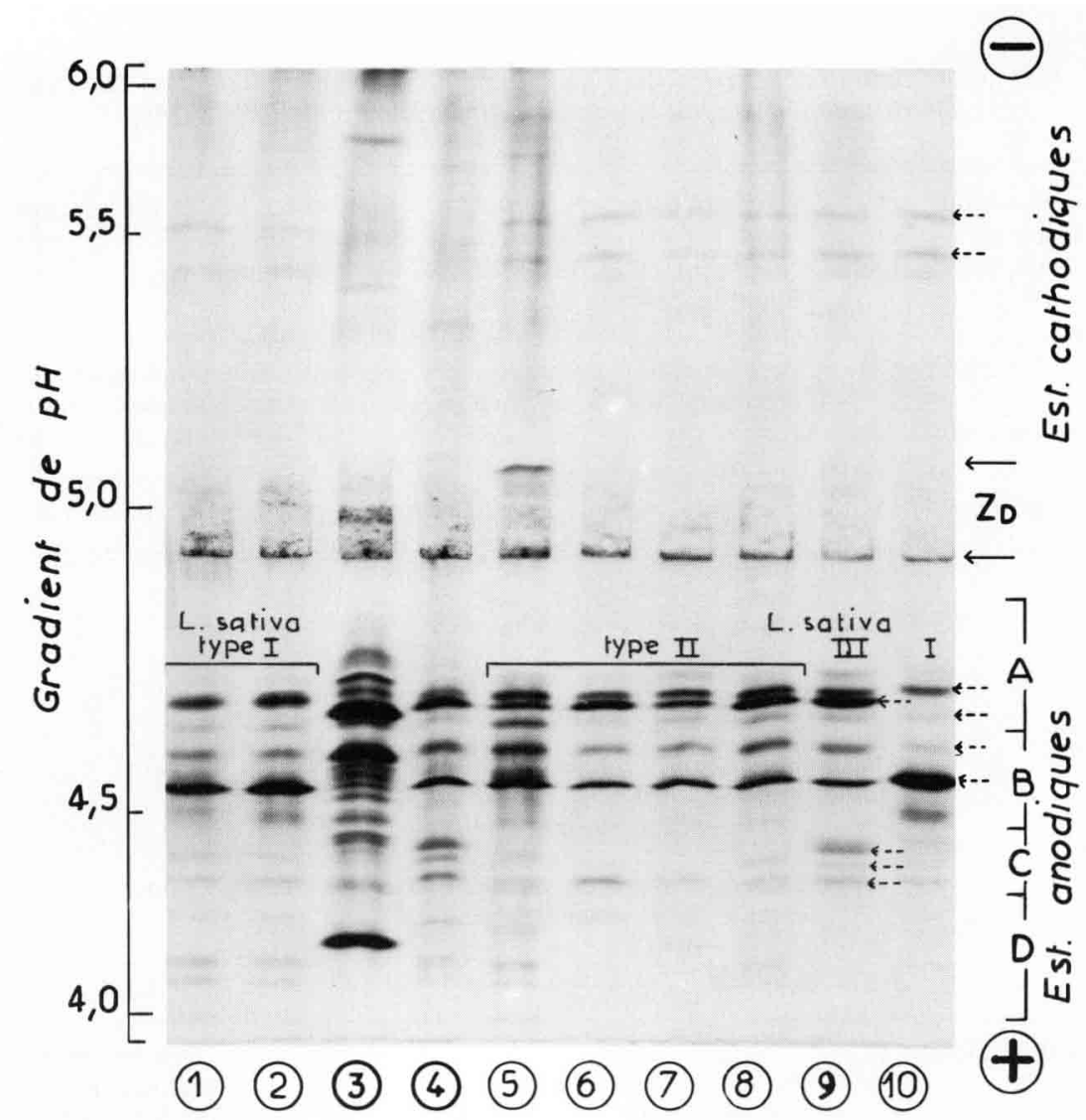

Figure 2

Diagrammes d'électrofocalisation (gradient de pH 4 à 6,5) des estérases foliaires de divers génotypes du genre Lactuca :

Electrofocusing patterns ( $\mathrm{pH}$ gradient from 4 to 6.5) of foliar esterases from different genotypes of the genus Lactuca.

L. sativa type I: (1) et (2) variétés chinoises; chinese cultivars; (10) "Novir".

L. sativa type II : (5) à (8) : "Rouge grenobloise », "Girelle », "Ondine ", "Calicel ».

déposés sur de petits rectangles de papier-filtre $(10 \times 5 \mathrm{~mm})$ disposés sur le gel à $5 \mathrm{~cm}$ de l'anode (fig. 2 à 6). Le tableau 2 fournit une vue d'ensemble des caractéristiques de la séparation, basée sur les instructions fournies par la firme LKB.

\section{E. Mise en évidence des estérases}

Dès la fin de l'électrofocalisation, le gel est plongé dans un milieu réactionnel dérivé de celui décrit par SHAW \& PRASAD (1970). $100 \mathrm{mg}$ de Fast Blue RR sont dissous par agitation à l'obscurité pendant 10 à $15 \mathrm{mn}$ dans $100 \mathrm{ml}$ de tampon Tris- $\mathrm{HCl} 1 \mathrm{M} \mathrm{pH} \mathrm{7,5;}$ $50 \mathrm{mg}$ d'alpha-naphtylacétate de sodium, préalablement dissous dans $3 \mathrm{ml}$ d'acétone, sont ajoutés juste avant l'emploi du réactif. On laisse la révélation se poursuivre pendant 20 à $40 \mathrm{mn}$ dans une étuve à $37{ }^{\circ} \mathrm{C}$. Le gel est ensuite rincé pendant $10 \mathrm{mn}$ à l'eau courante avant d'être plongé pendant $1 \mathrm{~h}$ dans un bain de fixation contenant : éthanol $25 \mathrm{ml}$, acide acétique concentré $8 \mathrm{ml}$, glycérol $10 \mathrm{ml}$, eau $67 \mathrm{ml}$. Après 15 à $20 \mathrm{~h}$ de séchage à température ambiante, le gel peut être recouvert d'une feuille de Mylar, ce qui permet de le conserver et de le manipuler aisément. La coloration obtenue est très stable.
L. sativa type III : (9): «Sucrine».

L. saligna (CRI7): (3); L. serriola (Courville) : 4 .

Les flèches indiquent les bandes retenues comme éléments descrip teurs caractéristiques de L. sativa (cf. fig. I).

Arrows indicate the bands retained as characteristic descriptors of L. sativa (cf. fig. 1 ).

$Z_{D}=$ zone de dépôt des échantillons; Zone for sample application.

\section{RÉSULTATS}

Une $1^{\text {re }}$ série de séparations, effectuée en gradient de $\mathrm{pH} 3,5$ à 9,5, avait fourni des diagrammes très denses dans la zone des points isoélectriques (pI) 4 à 6, difficiles à comparer entre eux. Cette zone étant celle qui fournit l'essentiel des caractères descriptifs, elle a été uniquement étudiée par électrofocalisation avec un gradient de $\mathrm{pH}$ de 4 à 6,5 qui assure une séparation plus distincte des bandes de $p I$ très voisins. Le pI d'une bande ne peut être mesuré avec une précision suffisante pour que cela représente un critère sûr d'identification : c'est la position relative des bandes par rapport à celles d'un ou plusieurs génotypes pris comme référence qui est déterminante pour leur identification. Les diagrammes obtenus pour les différents génotypes ont été confirmés par 4 à 8 déterminations portant sur des plantes distinctes.

Pour clarifier la description des diagrammes, la partie se situant entre la zone de dépôt des échantillons et l'anode a été divisée en 4 zones dénommées $\mathrm{A}, \mathrm{B}, \mathrm{C}$ et $D$. Le schéma de la figure 1 indique, pour chaque zone, les bandes retenues comme éléments descripteurs. Les bandes décrites n'ont pas reçu de dénomination particulière : seule l'étude des descendances 


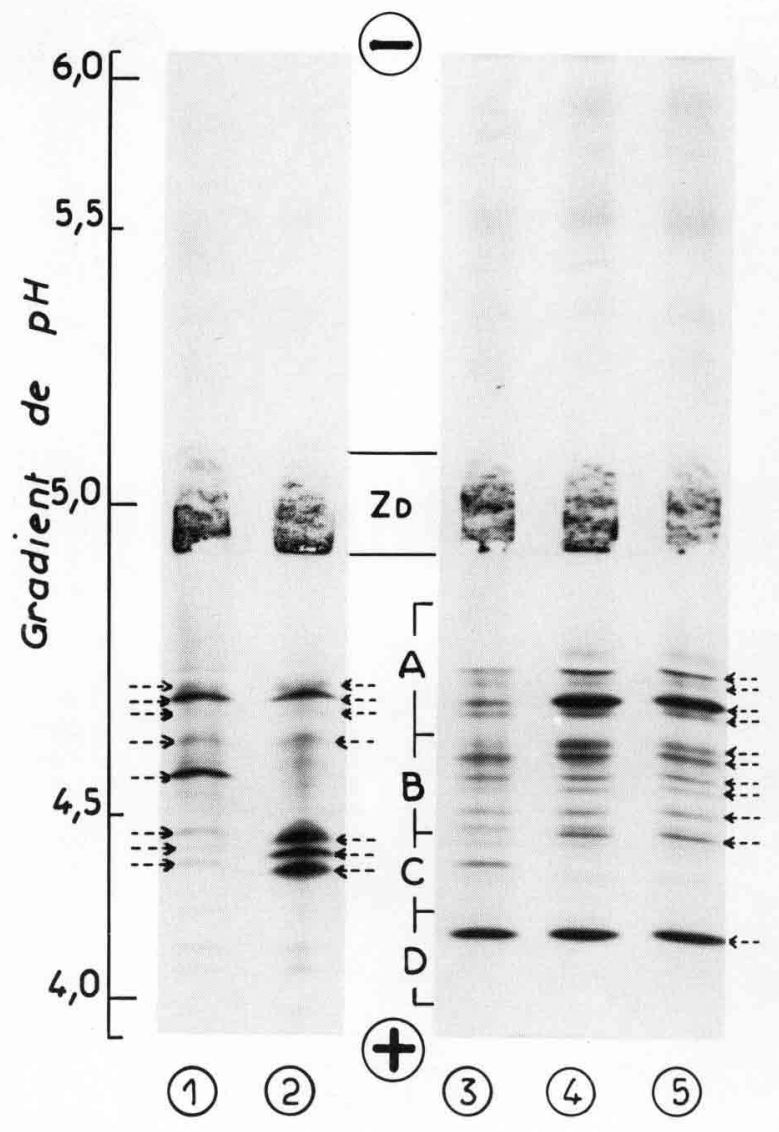

Figure 3

Diagrammes d'électrofocalisation (gradient de $p H 4$ à 6,5) des estérases foliaires chez divers génotypes du genre Lactuca.

Elecirofocusing patterns ( $\mathrm{pH}$ gradient from 4 to 6.5) of foliar esterases from different genotypes of the genus Lactuca.

(1) L. serriola (CR07) (2) L. aculeata (CR09)

(3), (4), (5) : L. saligna (CR10, $C R I 7, I V T 1346)$

Les flèches indiquent les bandes retenues comme éléments descripteurs caractéristiques (cf. fig. 1).

Arrows indicate the bands retained as characteristic descriptors (cf. fig. 1).

$Z_{D}=$ zone de dépôt des échantillons. Zone for sample application.

intra et interspécifiques permettra ultérieurement de préciser la signification de chacune d'elles (expression d'allèles, de un ou plusieurs loci...).

L'ensemble des variétés étudiées de L. sativa se répartit entre 3 types très voisins, ne différant qu'aux niveaux $A$ et $C$ (fig. 1 et 2).

$$
\begin{aligned}
& \begin{array}{ccccc}
\text { Type } & \text { Zone A } & \text { Zone B } & \text { Zone C } & \begin{array}{c}
\text { Variétés } \\
\text { correspondantes }
\end{array} \\
- & - & - & - & -
\end{array} \\
& \text { I } 2 \text { bandes } 2 \text { bandes - «BZ36» "Dorée de } \\
& \text { printemps ", " Illera ", } \\
& \text { " Melina ", " Novir ", } \\
& \text { « Serda », « Verpia », Va- } \\
& \text { riétés chinoises. } \\
& 3 \text { bandes }
\end{aligned}
$$

Les 6 génotypes de $L$. serriola sont semblables au type III de $L$. sativa. Ils s'en différencient seulement par le fait que 2 bandes faibles situées du côté de la cathode, uniformément présentes chez $L$. sativa, sont absentes ici (fig. 2). Un seul génotype de L. aculeata a pu être étudié (fig. 1 et 3). Son diagramme d'estérases anodiques se rapproche de celui de $L$. serriola, avec les particularités suivantes :

- au niveau B la bande d'intensité forte présente chez $L$. serriola est faible à inexistante,

- au niveau $\mathrm{C}$ apparaissent 3 bandes intenses dont il n'est pas possible de définir si elles ont ou non le même pI que les 3 bandes présentes chez $L$. serriola.

Les 6 génotypes de $L$. virosa présentent 2 bandes communes avec $L$. sativa; l'une au niveau A, l'autre au niveau B (fig. 1, 4 et 5). Une bande du niveau B est spécifique de $L$. virosa : cette bande est la même pour tous les génotypes sauf $L$. virosa forme maculata (IVT1398).

Les 3 génotypes de $L$. saligna se séparent nettement des autres espèces par des diagrammes plus complexes (fig. 1, 3 et 5). L'homologie possible avec les autres espèces se limite au niveau B qui présente peut-être une bande commune avec $L$. sativa et une avec L. virosa (fig. 1). Le génotype CR10 apparaît légère-
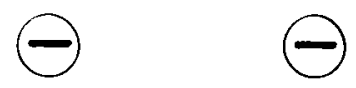
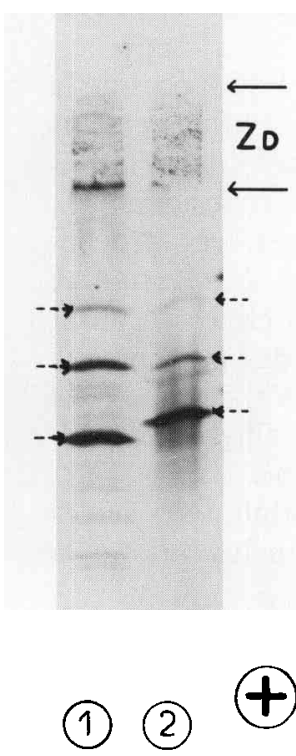

Fig. 4
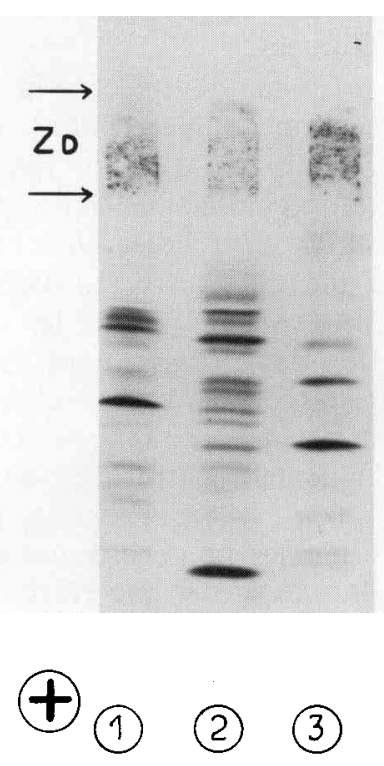

Figures 4 et 5

Diagrammes d'électrofocalisation (gradient de $p H 4$ à 6,5) des estérases foliaires chez divers génotypes du genre Lactuca.

Electrofocusing patterns ( $\mathrm{pH}$ gradient from 4 to 6.5) of foliar esterases from different genotypes of the genus Lactuca.

Fig. 4. (1) L. virosa (LS184); (2) L. virosa forme maculata (IVT1398)

Fig. 5. (1) L. sativa (Sucrine) ; (2) L. saligna (CR17) ; (3) L. virosa (LS238).

Les flèches indiquent les bandes retenues comme éléments descripteurs caractéristiques de L. virosa (cf. fig. I).

Arrows indicate the bands retained as characteristic descriptors of L. virosa (cf. fig. 1). 
ment différent de CR16 et CR17 (fig. 3) : en fait il s'agit surtout de différences d'intensité dans la révélation des bandes ; la particularité de CR 10 tient à une bande qui lui est spécifique au niveau $\mathrm{C}$. Au niveau $\mathrm{D}$, les génotypes de $L$. saligna présentent une bande intense très caractéristique.

Les diagrammes des hybrides $F 1, L$. sativa $\times L$. virosa, comportent l'ensemble des bandes présentes chez chacun des parents, ce qui les caractérise sans ambiguïté (fig. 6). N'ayant pas d'information sur le degré d'homologie des chromosomes de ces 2 espèces, on ne peut toutefois parler de codominance.

\section{DISCUSSION}

Pour chacune des espèces où l'on a pu étudier plusieurs génotypes ( $L$. sativa, $L$. serriola, $L$. virosa, L. saligna), on constate la faible variabilité du diagramme des estérases à l'intérieur de chaque espèce, quelle que soit son origine géographique. Chez L. sativa, la sélection a conduit à des types très variés de morphologie de l'appareil végétatif. Notamment les variétés d'origine chinoise proviennent d'une sélection qui s'est déroulée dans un contexte d'isolement (LINDQVIST, 1960b) et qui a donné naissance à un type très particulier : laitue à tige fortement tubérisée et à feuilles lancéolées. Or les 2 variétés d'origine chinoise présentent un diagramme d'estérases de type I, identique à celui de plusieurs variétés européennes. Cette faible variabilité intraspécifique des diagrammes d'isoenzymes des estérases permet de les considérer comme un bon critère de caractérisation des espèces étudiées.

En ce qui concerne la parenté entre les espèces de la section Lactuca (FERAKOVA, 1976), les renseignements fournis par le phénotype et par les possibilités d'hybridation interspécifique permettent de considérer $L$. sativa et $L$. serriola comme 2 espèces très proches l'une de l'autre (LINDQvisT, 1960a et $b$; RYDER \& WHITAKER, 1976) : les diagrammes d'électrofocalisation des estérases confirment ce point de vue. Lorsque les espèces sont plus éloignées entre elles, comme c'est le cas avec $L$. saligna et $L$. virosa qui s'hybrident difficilement entre elles ou avec $L$. sativa (LINDQVIST, $1960 a$; MAXON SMITH, 1984), la variabilité des seules estérases ne permet pas de tirer de conclusions sur la parenté génétique entre ces espèces.

Les croisements interspécifiques entre $L$. sativa et $L$. virosa ou $L$. saligna étant d'une réalisation difficile, le caractère hybride de la descendance demande à être vérifié. L'examen des diagrammes des estérases permet de tester l'état hybride des plantes obtenues en $F 1$, très tôt et sans perturber leur croissance. D'autre part, les hybrides F1 $L$. sativa $\times L$. virosa ont une fertilité très faible (LINDQVIST, 1960a; MAXON SMITH, 1984), que l'on peut accroître en produisant des amphidiploïdes par traitement de bourgeons axillaires à la colchicine. La confirmation de l'allopolyploïdie de la F2 peut être obtenue par comptage des chromosomes mais aussi par le biais des diagrammes des estérases qui sont alors tous homologues de ceux

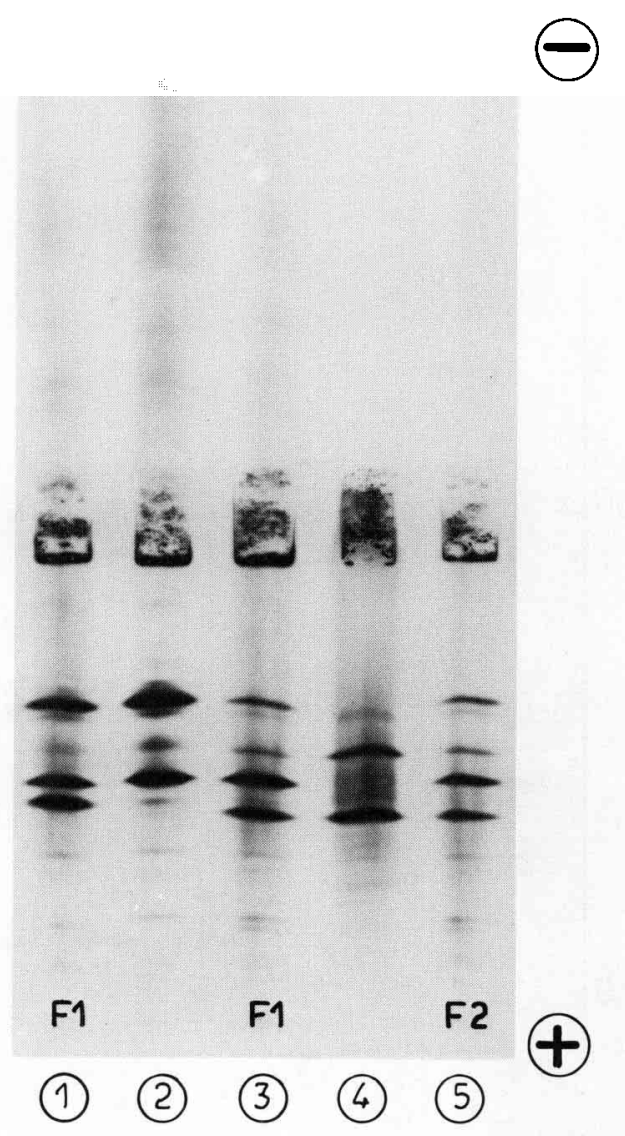

Figure 6

Diagramme d'électrofocalisation (gradient de $p H$ à 6,5) des estérases foliaires chez des hybrides L. sativa $(Q) \times$ L. virosa $(O)$.

Electrofocusing patterns ( $p H$ gradient from 4 to 6.5) of foliar esterases from hybrids L. sativa (Q) $\times$ L. virosa $\left(O^{\circ}\right)$.

(1) Hybride F1 L. sativa (Girelle mâle stérile) $\times$ L. virosa forme maculata (IVT1398)

(2) L. sativa (Girelle mâle stérile)

(3) Hybride F1 L. sativa (Girelle mâle stérile) $\times$ L. virosa (LSI84)

(4) L. virosa (LS184)

(5) F2 obtenue à la suite du traitement par la colchicine de l'hybride Fl décrit en (3) : toutes les plantes sont homologues des plantes Fl.

$F 2$ obtained after colchicine treatment of the F1 hybrid described in (3) : all the plants are homologous with the FI plants.

des plantes de la F1 (fig. 6). Lors de chacune des étapes ultérieures de la sélection (rétrocroisements), le choix des individus à conserver relève classiquement de 2 critères : la présence du caractère à introduire et un phénotype se rapprochant de celui du parent récurrent. En discernant parmi les individus retenus ceux qui se rapprochent le plus du parent récurrent du point de vue de l'expression des isoenzymes, on peut accélérer le processus de sélection proportionnellement au nombre de loci indépendants étudiés (TANKSLEY, 1983). Dans cette optique, l'étude de la variabilité d'autres systèmes enzymatiques va être poursuivie chez $L$. sativa et les espèces apparentées. 


\section{RÉFÉRENCES BIBLIOGRAPHIQUES}

Bassiri A., Adams M. W., 1978a. An electrophoretic survey of seedling isozymes in several Phaseolus species. Euphytica, 27, 447-459.

Bassiri A., Adams M. W., 1978b. Evaluation of common bean cultivars relationship by means of isozyme electrophoretic patterns. Euphytica, 27, 707-720.

Ferakova V., 1976. Lactuca, p. 328-331. In "Flora Europaea ", vol. 4, Cambridge University Press, $505 \mathrm{p}$.

Globerson D., Netzer D., Sacks J., 1980. Wild lettuce as a source for improving cultivated lettuce, 86-96. Proc. Eucarpia Meet. on leafy vegetables, Littlehampton, 11-14 March 1980. Ed. J. W. Maxon Smith \& F. A. Langton, Glasshouse Crop Research Institute, Littlehampton, UK., 137 p.

Gottlieb L. D., 1981. Electrophoretic evidence and plant populations, 1-46. In L. Reinhold et al.: " Progress in Phytochemistry", vol. 7. Pergamon Press, Oxford, $344 \mathrm{p}$.

Jensen U., 1981. Proteins in plant evolution and systematics, 344369. In H. Ellenberg et al. : "Progress in Botany », vol. 43. Springer-Verlag, Berlin-Heidelberg, $382 \mathrm{p}$.

Kahler A. L., Allard R. W., 1981. Worldwide patterns of genetic variation among four esterase loci in barley (Hordeum vulgare L.). Theor. Appl. Genet., 59, 101-111.

Lindqvist K., 1960a. Cytogenetic studies in the serriola group of Lactuca. Hereditas, 46, 75-151.

Lindqvist K., 1960b. On the origin of cultivated lettuce. Hereditas, 46, 319-350.

Maxon Smith J. W., 1984. Interspecific hybridization in Lactuca with particular reference to L. sativa L. $\times$ L. virosa L. Coll. Eucar- pia sur les légumes à feuilles, Versailles, 28 février-2 mars 1984. 21-34, Ed. H. Bannerot \& C. Meheut, Station de Génétique et d'Amélioration des plantes, I.N.R.A., Versailles, 206 p.

Moore G. A., Collins G. B., 1983. New challenges confronting plant breeders, 25-58. In S. D. Tanksley \& T. J. Orton : « Isozymes in plant genetics and breeding ». Part A. Elsevier, Amsterdam, $516 \mathrm{p}$.

Rhodes M. J. C., 1977. The extraction and purification of enzymes from plant tissues, 245-269. In H. Smith : « Regulation of enzyme synthesis and activity in higher plants ». Acad. Press, New York and London, $388 \mathrm{p}$.

Rodenburg C. M., 1960. Variétés de Laitues. Instit. vered. Tuinbouwg., Wageningen, Hollande, $228 \mathrm{p}$.

Ryder E. J., Whitaker T. W., 1976. Lettuce, 39-41. In N. W. Simmonds : "Evolution of crop plants ", Longman, London, 339 p.

Shaw C. R., Prasad R., 1970. Starch gel electrophoresis of enzymes. A compilation of recipes. Biochem. Genet., 4, 297-320.

Smith H. H., Hamill D. E., Weaver E. A., Thompson K. H., 1970. Multiple molecular forms of peroxidases and esterases among Nicotiana species and amphiploids. J. Hered., 61, 203-212.

Symeonidis L., Tsekos I., 1984. Electrophoretic variation in esterases, peroxidases and acid phosphatases in some native Greek taxa of the genera Hordeum and Taeniatherum. Ann. Bot., 53, 383-397.

Tanksley S. D., 1983. Introgression of genes from wild species, 331 337. In S. D. Tanksley \& T. J. Orton : « Isozymes in Plant Genetics and Breeding », Part A. Elsevier, Amsterdam, $516 \mathrm{p}$.

Tanksley S. D., Rick C. M., 1980. Genetics of esterases in species of Lycopersicon. Theor. Appl. Genet., 56, 209-219. 